\title{
AN IMPROVED METHOD FOR THE DETERMINATION OF PREGERMINATED GRAINS IN BARLEY
}

\author{
by \\ SVEND AAGE JENSEN and FLEMMING HELTVED \\ Department of Biotechnology, Carlsberg Research Laboratory \\ Gamle Carlsbergvej 10, DK-2500 Copenhagen Valby
}

Keywords: Enzyme activity, fluorescence, seed fixation

\begin{abstract}
A rapid and sensitive analysis for determining pregerminated grains in barley is described. The method is based upon a visualization of enzyme activity in longitudinally divided half seeds using the lipase sensitive fluorochrome, fluorescein dibutyrate. Parallel morphological studies of seed halves from the same pregerminated grain by the fluorescein dibutyrate method and by the established EBC-method (starch agar/iodine) revealed good coincidence between the two methods. The fluorescein dibutyrate test is faster, more sensitive and more reproducible as compared to the EBC-method.

Pregermination and germination analyses of barley seed lots with pregermination percentages of $0 \%$ to $46 \%$, showed a significant inverse relation between viability and pregermination percentage after 12 months storage in the laboratory. It is concluded that pregermination percentages as low as $2-3 \%$ could cause a significant reduction in viable barley seeds after storage.
\end{abstract}

\section{INTRODUCTION}

Pregermination or preharvest sprouting of barley can be defined as an uncontrolled physiological process which takes place in the ears, especially when the weather at harvest is warm and wet. High incidence of pregerminated grains may effect barley quality for malting, seed grain, feed and food. Pregerminated barley grains are less dense and as such may not remain submerged during steeping of the barley. Such grains are lost in the overflow from the steeping tanks. Embryos of heat dried pregerminated barley grains may loose their ability to germinate. This results in the presence of unmodified barley in the malting process and also renders the barley unsuitable as seed grain. Finally, pregerminated barleys are more susceptible to mould attack which can lead to 1) gushing in beer brewed from such derived malt or 2) a loss in the nutritional value of the barley as feed. It is therefore obvious that a mounting interest has emerged in the control of preharvest sprouting of barley $(2,10)$.

There are a number of methods which have been used for assessing pregermination in samples of barley. Morphological assessment of root 
and shoot growth is difficult and highly subjective and does not register incipient pregerminated grains because marginal kernels are impossible to detect by the naked eye. For this reason other more sensitive and specific methods have been introduced. Recently, a method for measuring $\alpha$-amylase has been tested by the European Brewery Convention Analysis Committee and is now recommended and published (3). The EBCmethod is based on the work of HUYGERS and VAN DER BEKEN (7) and SCHUR et al. (11) and involves detection of $\boldsymbol{\alpha}$-amylase activity from imprints of barley half grains on starch agar plates which are subsequently developed by iodine staining. The method takes about 2 hours to perform and its application for assessing pregerminated samples of barley on intake to the elevator is therefore limited. An alternative method for measuring $\alpha$-amylase in pregerminated barley involves release of a dye (bound to starch) by the action of $\alpha$-amylase (2) which is then determined spectrophotometrically. The test can be performed within $20 \mathrm{~min}$ but has the disadvantage that one highly pregerminated grain in 100 may effect the results more than e.g. 10 slightly pregerminated seeds. It is obvious that the first barley would be better for either malting or seed grain than the second.

A method which on the one hand satisfies the requirements for speed and on the other enables a study of the frequency of pregermination in seed populations is therefore recommended. In the present communication, the EBC-method is compared to a fluorescence technique based upon a rapid visualization of enzyme activity in individual barley half kernels using a lipase sensitive fluorochrome (9) and a recently described seed fixation system (6).

\section{MATERIALS AND METHODS}

\subsection{Plant material}

The plant material analysed consisted of the sixteen barley varieties included in the EBC spring barley trials "B", 1981 (4). The barley varieties were grown at two different Danish locations Hyldager and Tystofte and were kindly supplied by Lic.agro. J. LARSEN, Carlsberg Plant Breeding, and by Director J. RASMUSSEN, Tystofte Experimental Station. The barley samples were stored in paper bags at $22{ }^{\circ} \mathrm{C}$ in the laboratory and had a moisture content at $11 \%$ in balance with a relative humidity at $40 \%$.

\subsection{Pregermination determination}

\subsubsection{The EBC-method}

The procedure for determining pregerminated grains in barley which has been adapted by the EBC Analysis Committee (3) was used as the reference method.

\subsubsection{The fluorescein dibutyrate test (FDB-test)}

The method is based upon hydrolysis of nonfluorescent fluorescein dibutyrate to butyrate and highly fluorescent fluorescein, a reaction known to be preferentially catalyzed by lipase (5). Individual half kernels are stained at room temperature with a $1 \cdot 10^{-3} \mathrm{M}$-solution of fluorescein dibutyrate in $80 \% \mathrm{v} / \mathrm{v}$ aqueous ethanol according to a procedure previously described (9). The seed fixation system recently reported by HeLrved et al. (6) is used to obtain $4 \times 50$ longitudinally sanded half seeds on plastic plates from each barley sample thus providing assessment based upon 200 individual barley kernels (see section 3.2). Visualization of pregerminated barley grains (appearence of yellow fluorescence) was carried out in a Malt Modification Analyser equipped with a pregermination analysis attachment (Dept. of Biotechnology, Carlsberg Research Center, DK-2500 Valby, Copenhagen, Denmark). The results are expressed as percentages of pregerminated seeds.

\subsubsection{Image analysis}

In order to quantify the area of enzyme diffusion patterns as visualized by the FDB-test and by the EBC-method, an image analyser system (Imanco Quantimet 720, Cambridge Instruments, England) was used. Details and specifications of this system have previously been reported (1). The fluorescein dibutyrate stained longitudinal half seeds were examined in a Reichert polyvar microscope (Reichert, Austria) with custom made fluorescence optics (9). The modified area of the half seed kernel (yellow fluorescence part) was measured and expressed as percentage of the whole area of the half seed kernel. This term is in the following referred to as degree of pregermination. Determination of pregermination degree of half seeds analysed ac- 
cording to the EBC-method was carried out in a similar way except that photographs of the kernels were taken on a Kodak Ektachrome 400 ASA film and the diapositives placed in a macrophotography attachment to the image analyser and measured. The experiments include the following studies: 1) the FDB-test compared to the EBC-method, 2) the reproducibility of the FDBtest, and finally 3 ) the reproducibility of the EBC-method (see section 3.1). In each of the three experiments individual barley grains were selected from the Tystofte sample set. They were divided into two halves using a razor blade and both halves analysed as described.

\subsection{Germination percentage}

Germination percentage in the barley samples was determined as previously described (8) without the addition of hydrogen peroxide.

\section{RESULTS AND DISCUSSION}

\subsection{Morphological comparison between the} FDB-method and the EBC-method

The development patterns of hydrolytical enzymes in individual barley grains with varying degrees of pregermination were followed by examining longitudinal half seed sections in which the one half seed was stained with fluorescein dibutyrate while the other was analysed for $\alpha$ amylase by the EBC-method (Figure 1). In kernel (A) only a slight background fluorescence was observed by the FDB-method and the imprint on the starch agar gel was stained completely by iodine thus demonstrating a non-pregerminated kernel. Kernel (B) represents a sprouted kernel showing a typical intense yellow fluorescence in the endosperm adjacent to scutellum thus indicating the presence of Iuorescein dibutyrate splitting enzymes. In the EBC-test of the same kernel $\alpha$-amylase was visualized by the light noniodine stained zone. In the next following series of barley kernels with increasing degree of pregermination (C-F, Figure 1) the configuration of the area visualized by the FDB-test coincides with the diffusion area of $\alpha$-amylase analysed by the EBC-method. In slightly pregerminated barley seeds the area visualized by the FDB-test tends to exceed the corresponding area visualized by the EBC-method while in highly pregerminated barley seeds an opposite tenden- cy was observed (E and F, Figure 1). The pattern of hydrolase development in pregerminated barley seeds (Figure 1) visualized by the FDB-test corresponds well with a previous study of germinating barley seeds (9) in which fluorescein dibutyrate splitting enzymes appeared invariably and symmetrically in the scutellum in the early stages of germination and later diffused into the distal region of the endosperm.

In order to quantify the development patterns of the hydrolytical enzymes in question, the modified areas visualized by the FDB-test and by the EBC-method were compared. The degree of pregermination of 94 pregerminated barley seeds were measured using the Image Analyser System. A relatively low correlation $(r=0.77$, Figure 2) was obtained between the two methods. According to the EBC-method (3), the area of the colourless spot must be at least $1 / 2$ of the area of the whole grain in order to be considered as indicating a pregerminated grain. Using this criterion, 50 kernels out of the 94 kernels (Figure 2) are considered as being pregerminated. With the same standard the corresponding number based on the FDB-test is 17. The EBC-method includes a transferring of $\alpha$-amylase from the barley half seed to the starch agar gel carried out at $37{ }^{\circ} \mathrm{C}$ in $30 \mathrm{~min}$. Two different inference problems will occur by this procedure. Firstly, in incipient pregerminated barley kernels, the small amount of $\alpha$-amylase produced may not be transferred quantitatively if a perfect contact is not achieved between the hand cut half seed and the starch agar gel. Secondly, in highly pregerminated grains the $\alpha$-amylase content may have been raised to a level at which $\alpha$-amylase diffusion occurs in the starch agar gel. These problems do not exist by the FDB-method since this procedure is based on an in situ determination.

The low correlation obtained between the FDB-method and the EBC-method could be due to less precision in one of the two methods. Thus the reproducibility within each of the two methods was tested. In Figure 3 the area visualized by the FDB-method was measured parallelly in two half seeds of 36 pregerminated grains. The high correlation coefficient ( $r=0.96)$ obtained, confirms a high reproducibility of the FDB-method and independence of choice of the two half 
FLUORESCEIN

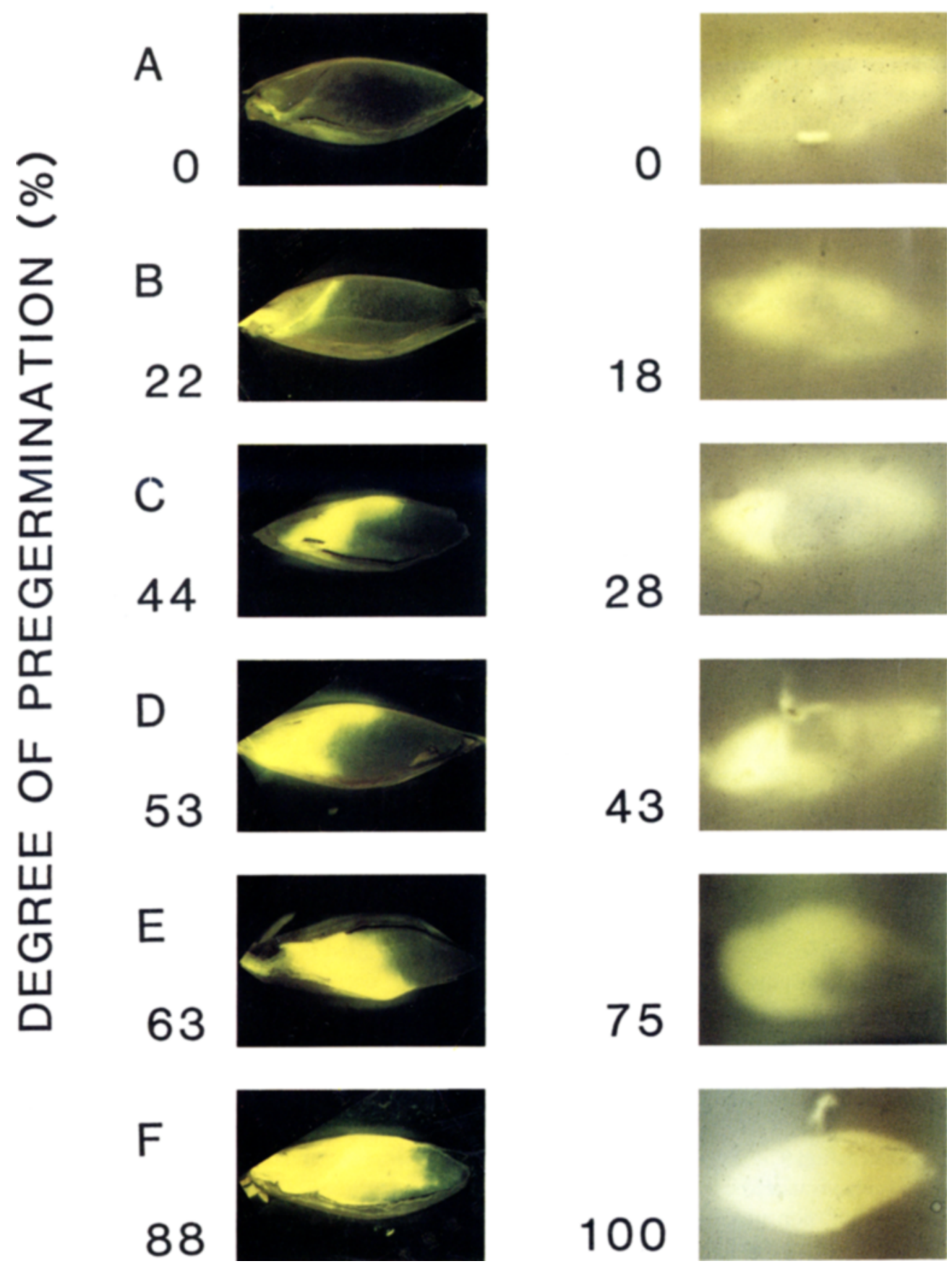

Figure 1. Varying degrees of pregermination in barley grains visualized, on the same seed (A-F) divided into two halves, by the FDB-method (left) and by the EBC-method (right).

The degrees of pregermination ranging from $0 \%$ to $88 \%$ (FDB-method) and from $0 \%$ to $100 \%$ (EBC-method) were measured by the Image Analyzer System. 

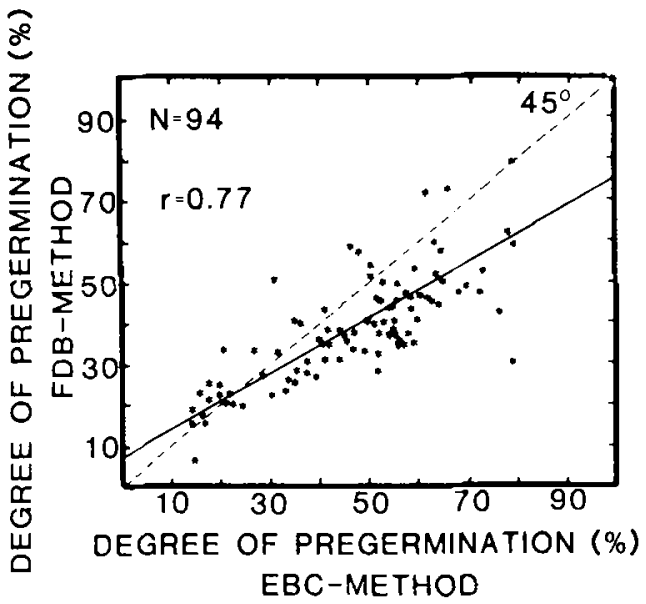

Figure 2. The relationship between the degree of pregermination determined by the FBC-method and by the FDB-method on longitudinally cut half seeds from the same seed.

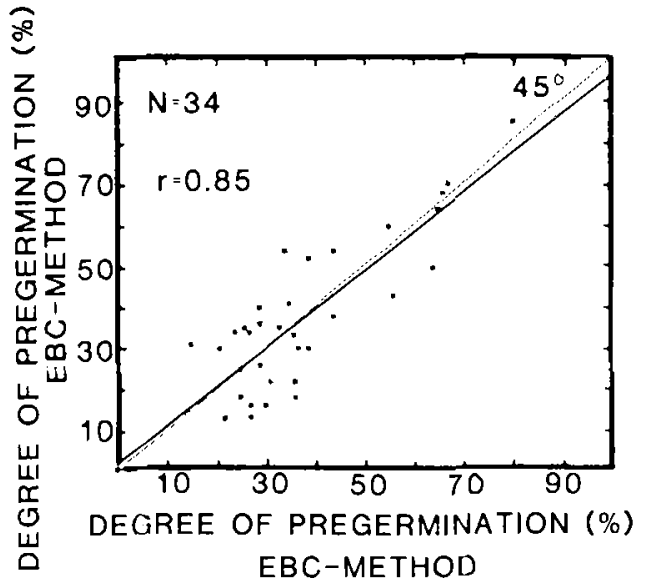

Figure 4. Reproducibility of the EBC-method determined on longitudinally cut half seeds from the same seed.

seeds. The corresponding results for the EBCmethod ( $N=34$, Figure 4 ) yielded a significantly lower correlation coefficient $(r=0.85)$. Thus the lower reproducibility of the EBC-method gives a reasonable explanation for the relatively low correlation observed between the two methods (Figure 2).

In the following study of determining the percentages of pregerminated grains in barley, the EBC-method was followed exactly and con-

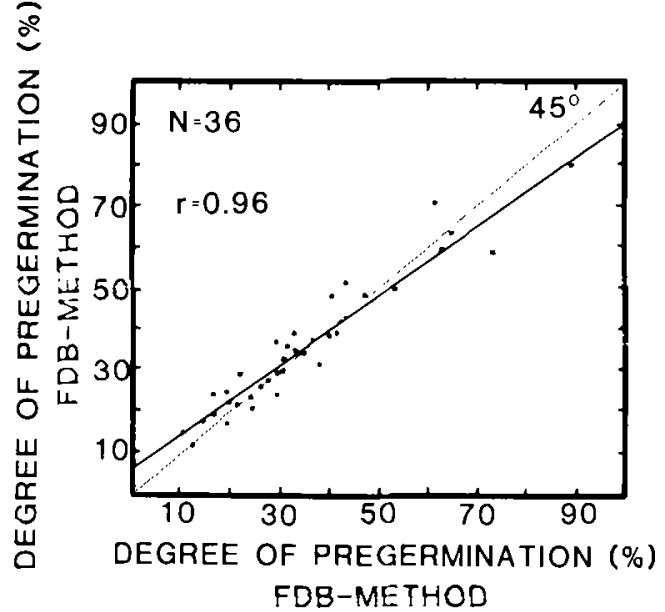

Figure 3. Reproducibility of the FDB-method determined on longitudinally cut half seeds from the same seed.

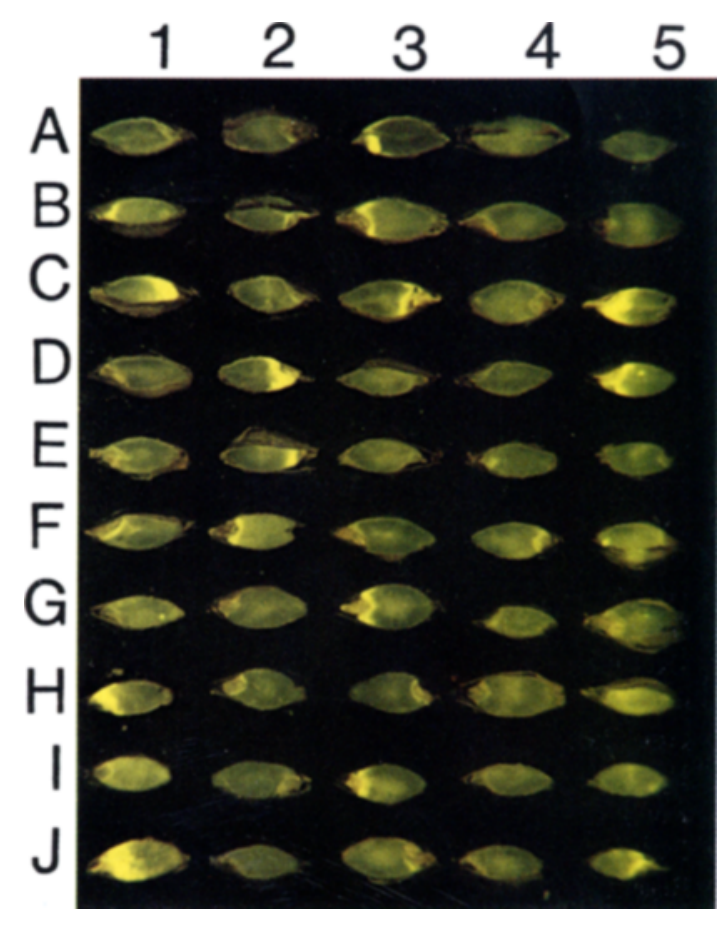

Figure 5. Photograph of 50 longitudinally sanded half seeds (variety Harry) stained with fluorescein dibutyrate and visualized in the Malt Modification Analyser equipped with a pregermination attachment.

$\mathrm{Cl}, \mathrm{C} 5, \mathrm{D} 2, \mathrm{D} 5, \mathrm{G} 3, \mathrm{H} 1$ and $\mathrm{J} 1$ have been judged highly pregerminated. $\mathrm{A} 3, \mathrm{~B} 1, \mathrm{~B} 3, \mathrm{C} 3, \mathrm{E} 2, \mathrm{~F} 1, \mathrm{~F} 2, \mathrm{~F} 4,13$ and $\mathrm{J} 5$ have been judged slightly pregerminated. 
Table I.

Pregermination and germination percentages of European Brewery Convention barley trials (serial B) grown at Tystofte 1981.

\begin{tabular}{|c|c|c|c|c|c|c|}
\hline \multirow[t]{3}{*}{ Variety } & \multicolumn{4}{|c|}{ Pregermination $\%$} & \multicolumn{2}{|c|}{ Germination \% } \\
\hline & \multicolumn{2}{|c|}{ Iodine test } & \multicolumn{2}{|c|}{ Fluorescein test } & \multirow{2}{*}{$\begin{array}{l}\text { Three wecks } \\
\text { after harvest } \\
\text { Sept. } 1981\end{array}$} & \multirow[t]{2}{*}{ Nov. 1982} \\
\hline & May 1982 & Nov. 1982 & May 1982 & Nov. 1982 & & \\
\hline Aramir & 2 & 5.5 & 2 & 0.5 & 95 & 82 \\
\hline Carina & 0 & 0 & 3 & 0 & 93 & 90 \\
\hline Beatrice & 0 & 0 & 0 & 0 & 51 & 95 \\
\hline Cerise & 0 & 0 & 2 & 3 & 97 & 89 \\
\hline Gimpel & 1 & 0 & 3 & 2 & 95 & 85 \\
\hline Harry & 20 & 13 & 46 & 28 & 93 & 16 \\
\hline Birka & 1 & 1 & 3 & 0.5 & 97 & 91 \\
\hline Cavrillon & 9 & 6 & 28 & 13 & 97 & 65 \\
\hline Carnival & 0 & 0 & 5 & 0 & 75 & 93 \\
\hline Gunhild & 0 & 0 & 8 & 0 & 97 & 98 \\
\hline Havila & 16 & 11.5 & 45 & 22.5 & 93 & 50 \\
\hline Koral & 0 & 0 & 0 & 0.5 & 83 & 97 \\
\hline Patty & 5 & 6 & 21 & 6 & 94 & 77 \\
\hline Regent & 1 & 2.5 & 15 & 6.5 & 88 & 87 \\
\hline Tintern & 0 & 0 & 0 & 0.5 & 97 & 97 \\
\hline Torkel & 3 & 4 & 14 & 3 & 96 & 79 \\
\hline
\end{tabular}

Table II.

Pregermination and germination percentages of European Brewery Convention barley trials (serial B) grown at Hyldager 1981.

\begin{tabular}{lcccc}
\hline Variety & \multicolumn{2}{c}{ Pregermination \% } & \multicolumn{2}{c}{ Germination \% } \\
& $\begin{array}{l}\text { Iodine test } \\
\text { Nov. 1982 }\end{array}$ & $\begin{array}{c}\text { Fluorescein test } \\
\text { Nov. 1982 }\end{array}$ & $\begin{array}{c}\text { Three weeks } \\
\text { after harvest } \\
\text { Sept. 1981 }\end{array}$ & Nov. 1982 \\
\hline Aramir & & & 91 & 98 \\
Carina & 0 & 0 & 91 & 99 \\
Beatrice & 0.5 & 0.5 & 42 & 99 \\
Cerise & 0 & 0.5 & 99 & 98 \\
Gimpel & 0 & 1 & 99 & 100 \\
Harry & 0 & 0.5 & 96 & 99 \\
Birka & 0 & 0.5 & 96 & 99 \\
Cavrillon & 0 & 0 & 91 & 99 \\
Carnival & 0 & 1 & 65 & 99 \\
Gunhild & 1 & 0 & 99 & 100 \\
Havila & 0.5 & 0 & 98 & 99 \\
Koral & 0.5 & 0.5 & 82 & 99 \\
Patty & 0.5 & 0.5 & 96 & 100 \\
Regent & 0 & 0 & 97 & 99 \\
Tintern & 0 & 0 & 97 & 99 \\
Torkel & 0 & 0 & 98 & 98 \\
\hline
\end{tabular}

stituted the reference method. For the FDBmethod, a barley grain was considered germinated when the appearance of a trace of intense yellow fluorescence was observed.

\subsection{Pregermination of barley}

The barley sample sets analysed for pregermination and germination percentages were the EBC barley trials (serial B 1981) grown at two 
different Danish locations, Tystofte (Table I) and Hyldager (Table II). In the Tystofte sample set both methods revealed a high pregermination frequence among the sixteen varieties. According to the EBC-method (analysed in May 1982) the variety Harry was observed to have the highest pregermination percentage $(20 \%)$ followed by Havila with $16 \%$ and Cavrillon with $9 \%$. These results were clearly reflected in the results obtained by the FDB-method (also analysed May 1982) though the latter method yielded a significantly higher germination percentage, e.g. Harry $46 \%$, Havila $45 \%$, and Cavrillon $28 \%$. This finding is, however, in good accordance with the more strict criterion of the FDB-method (see section 3.1). Figure 5 shows a photograph of 50 half seeds of Harry, stained with fluorescein dibutyrate and visualized in the Malt Modification Analyser. From this figure it can be seen that highly pregerminated barley grains are characterized by a large area of intense yellow fluorescence while incipient pregerminated barley grains are characterized by a small but sharp area of yellow fluorescence at the scutellum/endosperm interface. Non-pregerminated grains lack intense fluorescence.

It is noticeable that the same experiment performed six months later revealed a decrease in pregermination percentage (Table I, Nov. 1982) in both methods. These results indicate that the enzymes detected in both methods loose activity during storage of the grain.

Considering the germination percentages performed three weeks after harvest (Table I) thirteen varieties were acceptable in viability. Three varieties (Beatrice, Carnival and Koral) exhibited a lower germination percentage caused by a high degree of dormancy which could be eliminated by storage. The same experiment repeated a year later revealed an entirely different result (Table I). The samples with high pregermination percentages, e.g. Harry, Havila and Cavrillon had extremely low germination percentages (Harry 16\%, Havila $50 \%$ and Cavrilion $65 \%$ ), thus confirming the importance of a pregermination test as a quality parameter for barley determined for malting or seed grain. In general, a good inverse relationship exists between pregermination percentages and germination percentages after storage. The high level
(8\%) of pregerminated seeds which was obtained in one variety (Gunhild) as determined by the FDB-method in May 1982 was not repeated after storage and was not reflected by a low germination percentage. The discrepancy might be due to an experimental error.

Tuming to the other sample set grown at Hyldager (Table II) all sixteen varieties exhibited pregermination percentages of $1 \%$ or less as analysed by the EBC-method and by the FDB-method. The low incidence of pregermination was reflected in the high germination percentages ranging from $98 \%$ to $100 \%$ (Nov. 1982). The high degree of dormancy in Beatrice, Carnival and Koral was again seen in the germination percentages determined three weeks after harvest.

\section{CONCLUSION}

The FDB-method discussed in the present communication represents a sensitive and rapid analysis for the determination of pregerminated grains in barley. The test can be completed in less than $15 \mathrm{~min}$ and is thus an attractive alternative to the EBC-method, enabling a convenient control of pregerminated barley grains at the intake to the elevator. In addition the FDB-method is more sensitive in detecting incipient preharvest sprouting than the EBC-method.

The inverse relation observed between percentages of pregerminated barley grains and germination percentages in the EBC barley sets (after storage) clearly confirmed that a low pregermination figure for barley is important to retain a good storage quality. In the present experiment the pregermination percentage of a barley sample as analysed by the FDB-method should not exceed $2-3 \%$ in order to avoid significant losses due to non-viable seeds after storage. The present experiments suggest that both varietal and environmental factors significantly influence the degree of pregermination. The FDBmethod could be instrumental in exploring such complex interactions with regard to viability in storage.

\section{ACKNOWLEDGEMENTS}

We wish to thank Fil. Dr. LARS MUNCK and Dr. Gregory Gibbons for stimulating discussions and for reviewing the manuscript. The assistance 
of ANNETTE HANSEN for preparing the figures and of $\mathrm{K}$. KIRKEGAARD for typing the manuscript is gratefully acknowledged.

\section{REFERENCES}

1. AASTRUP, S. \& K. ERDAL: Quantitative determination of endosperm modification and its relationship to the content of $1,3: 1,4$ - $\beta$-glucans during malting of barley. Carlsberg Res. Commun. 45, 369-379 (1980)

2. BROOKES, P.A.: The significance of preharvest sprouting of barley in malting and brewing. Cereal Research Commun. 8, 29-38 (1979)

3. European Brewery Convention. Analytica III. Method 2.6A, Zoeterwoude, The Netherlands.

4. European Brewery Convention. Report of the Barley Committee, field trials 1981, Vol. 32. EBC, Zoeterwoude, The Netherlands

5. Guilbault, G.G. \& D.N. Kramer: Fluorometric determination of lipase, acylase, alfaand gamma-chymotrypsin and inhibitors of these enzymes. Anal. Chem. 36, 409-412 (1964)
6. Heltved, F., S. Aastrup, O. Jensen, G. GibBONS \& L. MuNCK: Preparation of seeds for mass screening. Carlsberg Res. Commun. 47, 291-296 (1982)

7. Huygers, R. \& R. VAN DER Beken: Le Petit Journal du Brasseur. 84, 346 (1976).(Referred to in European Brewery Convention. Analytica III. Method 2.6A)

8. International Seed Testing Association: International rules for seed testing. Seed Sci. Technol. 4, 41-177 (1976)

9. Jensen, S.AA. \& F. Heltved: Visualization of enzyme activity in germinating cereal seeds using a lipase sensitive fluorochrome. Carlsberg Res. Commun. 47, 297-303 (1982)

10. Lloyd, W.J.W. \& P.A. Martin: The determination of germinated grains in barley. J. Inst. Brew. 88, 322-323 (1982)

11. Schur, F., P. Anderegg \& H. Pfenninger: Alfa-amylasen in Gerste, Malz und microbiellen Enzympräparaten. Schweizer BrauereiRundschau, 88 (1), 3-9 (1977) 